Old Dominion University

ODU Digital Commons

1983

\title{
On the First Passage Time Distribution for a Class of Markov Chains
}

Mark Brown

Narasinga Rao Chaganty

Old Dominion University, rchagant@odu.edu

Follow this and additional works at: https://digitalcommons.odu.edu/mathstat_fac_pubs

Part of the Probability Commons, and the Set Theory Commons

\section{Original Publication Citation}

Brown, M., \& Chaganty, N. R. (1983). On the first passage time distribution for a class of Markov chains. Annals of Probability, 11(4), 1000-1008. doi:10.1214/aop/1176993448

This Article is brought to you for free and open access by the Mathematics \& Statistics at ODU Digital Commons. It has been accepted for inclusion in Mathematics \& Statistics Faculty Publications by an authorized administrator of ODU Digital Commons. For more information, please contact digitalcommons@odu.edu. 


\title{
ON THE FIRST PASSAGE TIME DISTRIBUTION FOR A CLASS OF MARKOV CHAINS
}

\author{
By Mark Brown ${ }^{1}$ and Narasinga R. Chaganty \\ City College, CUNY and Old Dominion University
}

\begin{abstract}
Consider a stochastically monotone chain with monotone paths on a partially ordered countable set $S$. Let $C$ be an increasing subset of $S$ with finite complement. Then the first passage time from $i \in S$ to $C$ is shown to be IFRA (increasing failure rate on the, average). Several applications are presented including coherent systems, shock models, and convolutions of IFRA distributions.
\end{abstract}

1. Introduction. Let $S$ be a countable set with a partial ordering denoted by $\leq$. Consider a discrete time Markov chain $\left\{X_{n}, n \geq 0\right\}$ with state space $S$, and transition matrix $P$. Define the Markov chain to have monotone paths if $\operatorname{Pr}\left(X_{n+1} \geq X_{n}\right)=1$. Define $C \subset S$ to be an increasing set if $i \in C$ and $j \geq i$ implies $j \in C$. Define the Markov chain to be stochastically monotone if $i \leq j$ implies $P(i, C) \leq P(j, C)$ for all increasing sets $C$.

For a state $i$ and set $C$ define $T(i, C)$ to be the first passage time from $i$ to $C$, with $T(i, C)=0$ if $i \in C$, and $T(i, C)=\infty$ if $C$ is never reached. Our main result (Theorem 1 ) is that for a stochastically monotone Markov chain with monotone paths on a partially ordered countable set, $T(i, C)$ is IFRA for all states $i$ and all increasing sets $C$ with finite complement. (See Section 2 for a definition of IFRA).

Conversely, every discrete IFRA distribution is either a first passage time distribution of the above described type or the limit of a sequence of such distributions (Corollary 1).

Several applications of Theorem 1 are presented in Section 5. These include coherent systems, shock models, convolutions of IFRA distributions, multinomial distributions, and sampling. In these applications $S$ is a subset of $R^{n}$ and the partial ordering is defined by $\mathbf{x} \leq \mathbf{y}$ if and only if $x_{i} \leq y_{i}, i=1, \cdots, n$. The possibility exists for applications to a wider class of partially ordered sets, for example those studied in combinatorial theory (Rota [14]).

The question arises as to whether the conclusion of Theorem 1 can be strengthened from IFRA to IFR. An example is given to show that even for a totally ordered set the first passage time need not be IFR. However for a Markov chain on the positive integers with $T P_{2}$ transition matrix the first passage time from 1 to $\{i: i>n\}, n=1,2, \cdots$ is IFR. This is proved in Section 6.2.

Examples are given which demonstrate that even for a totally ordered set the IFRA conclusion does not follow for a stochastically monotone chain without monotone paths nor for a non-stochastically monotone chain with monotone paths.

By a uniformization and total positivity argument the result (Theorem 1) extends to continuous time Markov chains with countable state space. An analogue of Theorem 1 undoubtedly holds for continuous state space. The restriction that the complement of $C$ be finite appears to be a limitation of our methodology rather than an essential condition.

2. Main result. A random variable $Y$ taking values in $\{0,1, \ldots, \infty\}$ is defined to be IFRA if either $\operatorname{Pr}(Y=0)=1$, or $\operatorname{Pr}(Y=0)=0$ and $[\operatorname{Pr}(Y>k)]^{1 / k}$ is decreasing in $k=1$,

Received August 1980; revised February 1983.

${ }^{1}$ Research supported by Air Force Office of Scientific Research Grant Nos. AFOSR F49620-79-C0157 and AFOSR 78-3678. This research was performed while Mark Brown was Professor of Statistics at Florida State University.

AMS 1980 subject classifications. Primary, 60J10; secondary, 60K10.

Key words and phrases. Markov chains, first passage times, reliability coherent systems, shock models, multinomial distributions, stochastic monotonicity, partially ordered sets, total positivity, IFRA, IFR, NBU. 
$2, \ldots$ Included as IFRA is the case $\operatorname{Pr}(Y=\infty)=1$. Note that if $Y$ is IFRA and $\operatorname{Pr}(Y<\infty)>0$ then $\operatorname{Pr}(Y<\infty)=1$.

Thus if $T(i, C)$ is IFRA then either $i \in C$ (in which case $T(i, C) \equiv 0$ ), or starting in $i$ it is impossible to reach $C$ (in which case $T(i, C) \equiv \infty)$, or $\operatorname{Pr}(T(i, C)=0)=\operatorname{Pr}(T(i, C)=\infty)$ $=0$ and $[\operatorname{Pr}(T(i, C)>k)]^{1 / k}$ is decreasing.

A real-valued function $f$ on a partially ordered set $D$ is defined to be decreasing if $d_{1} \geq d_{2}$ implies $f\left(d_{1}\right) \leq f\left(d_{2}\right)$.

Lemma 1 below is a rather trivial result, but for lack of a reference we include a proof in the appendix.

LEMмA 1. Let $f$ be a decreasing real-valued function on a finite partially ordered set $D$. Then the points in $D$ can be labeled in such a way that $f\left(d_{1}\right) \leq f\left(d_{2}\right) \cdots \leq f\left(d_{m}\right)$, and for $i<j, d_{i} \not \leq d_{j}$ ( $d_{i}$ is not smaller than $d_{j}$ under the partial ordering).

THEOREM 1. Let $\left\{X_{n}, n \geq 0\right\}$ be a stochastically monotone Markov chain with monotone paths on the partially ordered countable set $S$. Let $C$ be an increasing set with $\widetilde{C}$ (the complement of $C$ in $S$ ) finite. Then $T(i, C)$, the first passage time from state $i$ to set $C$, is IFRA.

Proof. Define $\bar{F}_{k}(i, C)=\operatorname{Pr}(T(i, C)>k)$. Our goal is to prove that $\left[\overline{\bar{F}}_{k}(i, C)\right]^{1 / k}$ is decreasing, equivalently that:

$$
\left[\bar{F}_{k}(i, C)\right]^{k+1} \geq\left[\bar{F}_{k+1}(i, C)\right]^{k}, \quad k=1,2, \cdots .
$$

The proof is by induction. We first show that (1) holds for $k=1$. It is trivially true for $i \in C$. For $i \notin C$ by the monotone path assumption:

$$
\bar{F}_{2}(i, C)=\sum_{b \geq i, b \in \tilde{C}} P(i, b) P(b, \tilde{C}) .
$$

By stochastic monotonicity $b \geq i$ implies $P(i, \widetilde{C}) \geq P(b, \widetilde{C})$, thus from (2):

$$
\bar{F}_{2}(i, C) \leq P(i, \tilde{C}) \sum_{b \geq i, b \in \tilde{C}} P(i, b)=\left(\bar{F}_{1}(i, \tilde{C})\right)^{2} .
$$

Assume now that (1) holds for $k=1, \cdots, l$, for all increasing sets with finite complement. Define $P_{k}$ to be the $k$ step transition matrix for the Markov chain. Now:

$$
\left(\bar{F}_{l+1}(i, C)\right)^{l+2}=\bar{F}_{l+1}(i, C)\left[\sum_{b \in \tilde{C}} P_{l}(i, b) P(b, \tilde{C})\right]^{l+1} .
$$

By stochastic monotonicity $P(b, \widetilde{C})$ is decreasing in $b$. Since $\widetilde{C}$ is finite, it follows from Lemma 1 that we can label the finitely many points in $\widetilde{C}$ by $b_{1}, \cdots, b_{m}$ in such a way that $P\left(b_{1}, \widetilde{C}\right) \leq P\left(b_{2}, \widetilde{C}\right) \leq \cdots \leq P\left(b_{m}, \widetilde{C}\right)$ and for $i<j, b_{i} \not b_{j}\left(b_{i}\right.$ is not smaller than $b_{j}$ under the partial ordering). It follows that the sets $D_{0}=\widetilde{C}$, and $D_{j}=\widetilde{C}-\left\{b_{1}, \cdots, b_{j}\right\}, j=1$, $\ldots, m-1$, are finite sets which are complements of increasing sets. The induction hypothesis is thus applicable to these sets. Define $\alpha_{1}=P\left(b_{1}, \widetilde{C}\right)$ and $\alpha_{j}=P\left(b_{j}, \widetilde{C}\right)-$ $P\left(b_{j-1}, \tilde{C}\right), j=2, \cdots, m$, and note that the $b$ 's have been labeled so that:

$$
\alpha_{j} \geq 0, \quad j=1, \cdots, m \text {. }
$$

Next:

$$
\bar{F}_{l+1}(i, C)=\sum_{r=1}^{m} P_{l}\left(i, b_{r}\right) \sum_{j=1}^{r} \alpha_{j}=\sum_{j=1}^{m} \alpha_{j} P_{l}\left(i, D_{j-1}\right) .
$$

By the induction hypothesis:

$$
P_{l}\left(i, D_{j-1}\right) \geq\left[P_{l+1}\left(i, D_{j-1}\right)\right]^{l /(l+1)}, \quad j=1, \cdots, m .
$$

Note that in (7) if $i \notin D_{j-1}$, then both sides equal zero, and (7) is valid.

By (4), (5), (6) and (7):

$$
\left[\bar{F}_{l+1}(i, C)\right]^{l+2} \geq\left[\left(\bar{F}_{l+1}(i, C)\right)^{1 /(l+1)} \sum_{j=1}^{m} \alpha_{j}\left(P_{l+1}\left(i, D_{j-1}\right)\right)^{l /(l+1)}\right]^{l+1} .
$$


But, $\widetilde{C} \supset D_{j-1}$; by this fact and the monotone path assumption:

$$
\bar{F}_{l+1}(i, C)=P_{l+1}(i, \tilde{C}) \geq P_{l+1}\left(i, D_{j-1}\right), \quad j=1, \cdots, m .
$$

The result now follows from (6) (applied to $l+1),(8)$ and (9). $\square$

3. Continuous time. Consider a stochastically monotone Markov chain with monotone paths and countable state space. Suppose that changes of state occur according to a Poisson process with rate $\lambda$. Define $T^{*}(i, C)$ to be the first passage time (in continuous time) from state $i$ to $C$, an increasing set with finite complement. Now:

$$
\operatorname{Pr}\left(T^{*}(i, C)>t\right)=\sum_{k=0}^{\infty} \frac{(\lambda t)^{k} e^{-\lambda t}}{k !} \operatorname{Pr}(T(i, C)>k)
$$

By Theorem 1 and Theorem 3.6, page 93 of Barlow and Proschan [1], $T^{*}(i, C)$ is IFRA.

Next, consider a continuous time Markov chain with infinitesimal matrix $A$ taking values in a partially ordered countable set. Assume that the Markov chain has monotone paths, equivalently that $A(i, j) \neq 0$ implies $i \leq j$. Assume that the process is uniformizable, i.e. that $\sup (-A(i, i))<\infty$. Finally, assume that $A(i, C)=\sum_{l \in C} A(i, l) \leq A(j, C)$ for all increasing sets $C$, and $i \leq j$ with both $i$ and $j$ in $\widetilde{C}$.

By Cinlar [4] page 260, the Markov process is representable as a Markov chain with transition matrix $P=I+A / \lambda$ for which transitions occur according to a Pois̀son process of rate $\lambda$. The Markov chain with transition matrix $P$ inherits monotone paths. In view of monotone paths, the condition $A(i, C)=\sum_{l \in C} A(i, l) \leq A(j, C)$ for all increasing sets $C$ and $i \leq j$ with both $i$ and $j$ in $\widetilde{C}$ insures that the Markov chain is stochastically monotone. It follows from Theorem 1 and the remarks at the beginning of this section that if $C$ is an increasing set with finite complement then the first passage time to $C$ for the continuous time process is IFRA. To summarize:

Corollary 1. (i) Consider a Markov chain of the type described in Theorem 1. Suppose that transitions occur according to a Poisson process. Then the first passage time in continuous time from $i \notin C$ to $C$, an increasing set with finite complement, is IFRA.

(ii) Consider a Markov chain in continuous time with countable partially ordered state space and infinitesimal matrix A. Assume that:

(i) $\sup (-A(i, i))<\infty$

(ii) $A(i, j) \neq 0$ implies $i \leq j$

(iii) $A(i, C) \leq A(j, C)$ for all increasing sets $C$ with finite complement, and pairs of states $(i, j)$ with $i \leq j$ and $i, j$ both in $\widetilde{C}$.

Then the first passage time from $i \notin C$ to $C$, an increasing set with finite complement, is IFRA.

4. Converse. We will reinterpret a result of Birnbaum, Esary, and Marshall [2] (described in Barlow and Proschan [1], Theorem 2.13, page 88) in terms of Markov chains. The analogue of their result in the discrete case provides a converse to Theorem 1 .

The result is that every IFRA distribution in continuous time is either the system life for a coherent system of independent exponential components, or is the limit distribution of a sequence of such system lives. But a coherent system of $n$ independent exponential components is a continuous time Markov process on the set of the $2^{n} n$-tuples of 0 's and 1's with partial ordering $\mathbf{x} \leq \mathbf{y}$ if and only if $x_{i} \leq y_{i}, i=1, \cdots, n$. Moreover the process is stochastically monotone with monotone paths and the system lifetime is the first passage time to an increasing set. Thus every IFRA distribution in continuous time is either a first passage time distribution of the above described type or the limit of a sequence of such first passage time distributions.

Replacing continuous time by discrete time and exponential distributions by geometric distributions, we can imitate their proof and obtain the following result. 
LEMMA 2. Every IFRA distribution in discrete time is either the first passage time distribution to an increasing set for a stochastically monotone Markov chain with monotone paths on a partially ordered finite set, or the limit of a sequence of such distributions.

\section{Applications.}

5.1 Coherent systems. Consider a coherent system of $n$ independent components (Barlow and Proschan [1], Chapter 1, Section 2). Assume that the survival function for component $i$ is given by $(\bar{F}(t))^{\lambda_{t}}, i=1, \cdots, n$ where $F$ is continuous. This is known as the proportional hazard assumption. Each component starts off in state 0 , remains in 0 until failure, at which time it switches to state 1 and stays there forever. The system fails as soon as the state vector visits $B$, an increasing set. In this case $S$ is the set of $2^{n} n$-tuples of 0 's and 1's with partial order $\mathbf{x} \leq \mathbf{y} \Leftrightarrow x_{i} \leq y_{i}, i=1, \cdots, n$.

Consider the embedded discrete time process, starting in state $(0, \cdots, 0)$, and changing state each time a component fails. Under the proportional hazard assumption this process is a Markov chain. If the set of working components at a given instant is $A$, then given that a change of state occurs, the probability that component $i \in A$ failed is given by $\lambda_{i} / \sum_{A} \lambda_{j}$. The Markov chain is easily seen to be stochastically monotone with monotone paths. Therefore by Theorem 1 the number of component failures until system failure is IFRA. This generalizes a result of Ross, Shashahani, and Weiss [13], who proved the IFRA property in the case of i.i.d. components with continuous distribution $\left(\lambda_{i} \equiv \lambda\right)$.

By Corollary 1, if the component lifetimes are exponential then the time to first failure for the continuous time process is IFRA. This is a special case of the IFRA closure theorem (Barlow and Proschan [1] page 85).

5.2. Shock models. Assume that $Y_{1}, Y_{2}, \ldots$ are i.i.d. vectors of dimension $k$ and of arithmetic type. The set $\mathscr{S}$ consists of vectors of the form $\left(\lambda_{1} w_{1}, \cdots, \lambda_{k} w_{k}\right)$ where $w=$ $\left(w_{1}, \ldots, w_{k}\right)$ is a fixed vector of positive numbers and the $\lambda$ 's are non-negative integer valued. Define $S_{n}=\sum_{1}^{n} Y_{i}, n=1,2, \ldots$. Then $\left\{S_{n}, n \geq 1\right\}$ is a stochastically monotone Markov chain with monotone paths, and Theorem 1 is applicable. Let $g$ be a function, $R_{+}^{k}$ $\rightarrow R_{+}=[0, \infty)$, which is increasing in each argument and which goes to $\infty$ as $x_{i} \rightarrow \infty$ with $\left(x_{1}, \cdots, x_{i-1}, x_{i+1}, \cdots, x_{k}\right)$ held fixed, for $i=1, \cdots, k$. Then the first $n$ such that $g\left(S_{n}\right)$ exceeds a given constant $\gamma$ is IFRA. This is true because the set $\{\mathbf{x}: g(\mathbf{x})>\gamma\}$ is an increasing set with finite complement.

Interpreting $Y_{i}$ as the joint damage to $k$ components due to the $i$ th shock, $g$ as the rule for converting component damage into system damage, and $\gamma$ as the damage threshold beyond which the system fails, it follows that the number of shocks required for system failure is IFRA.

More generally the damage caused by a shock may depend on the previously accumulated damage. As long as damage is non-negative and the Markov chain remains stochastically monotone, the IFRA conclusion will hold.

If shocks occur in time according to a Poisson process then by Corollary 1 the waiting time (in continuous time) until system failure is IFRA.

Finally if the damages are not of arithmetic type, i.e. concentrated on a set $\mathscr{S}$ as described above, but are still componentwise non-negative, then by passage to the limit through approximating vectors of arithmetic type it follows that the time to system failure is IFRA. This is true for the discrete time process and for the continuous time process when shocks occur according to a Poisson process.

Thus the classic shock model results of Esary, Marshall, and Proschan [7] are derivable from Theorem 1 and Corollary 1. Moreover the current results are more general in that they allow for vector valued component damages and general system damage function $g$.

5.3. Multinomial distribution. Suppose that $Z_{1}, Z_{2}, \cdots$ are i.i.d. with $\operatorname{Pr}(Z=j)=p_{j}$, 


$$
\begin{aligned}
& j=1, \cdots, k, \sum_{1}^{k} p_{j}=1 \text {. Define } \\
& \qquad Y_{i j}=\left\{\begin{array}{lll}
1 & \text { if } & Z_{i}=j \\
0 & \text { if } & Z_{i} \neq j
\end{array}, \quad S_{n j}=\sum_{1}^{n} Y_{i j}, \quad \text { and } S_{n}=\left(S_{n 1}, \cdots, S_{n k}\right) .\right.
\end{aligned}
$$

The random variable $S_{n j}$ is the number of times that $j$ appears among $Z_{1}, \cdots, Z_{n}$, and $S_{n}$ is thus multinomially distributed. The Markov chain $\left\{S_{n}, n \geq 1\right\}$ is of the type considered in Section (5.2), the partial sums of i.i.d. random vectors with arithmetic distribution.

It follows that the number of observations required until at least $r$ categories each appear at least $m$ times is IFRA. The famous birthday problem (Feller [8] page 31) is an application with $r=1$ and $m=2$. For any distribution of births over the days of the year, the number of people sampled until two have the same birthday is IFRA.

5.4. Sampling with or without replacement. Sampling with replacement is covered by 5.3 above. For sampling without replacement, suppose that an urn contains $m_{i}$ balls of color $i, i=1, \cdots, k$. Then the following random variables are IFRA. The number of balls sampled until all balls of at least one color are depleted; until at least one ball of each color is sampled; until at least $r_{i}$ balls of type $i, i=1, \cdots, k$ are sampled; until at least $l$ of the events $A_{1}, \cdots, A_{k}$ occur, where $A_{i}=$ at least $r_{i}$ balls of type $i$ are sampled . The last case includes the first three.

5.5. Convolutions. The closure of IFRA distributions under convolutions was proved by Block and Savits [3]. We now present a different proof of this result.

Consider, first, IFRA distributions in continuous time. By the result of Birnbaum, Esary and Marshall [2], discussed in Section 4, it suffices to show that the convolution of two IFRA distributions, each of which is the system life for a coherent system of independent exponential components, is IFRA.

Let $T_{1}\left(T_{2}\right)$ be the system life for a coherent system of $n(m)$ independent exponential components with failure rates $\lambda_{1}, \cdots, \lambda_{n}\left(\mu_{1}, \cdots, \mu_{m}\right)$. For each system define the state at time $t$ to be the set of failed components at time $t$. The state space for system 1(2) is $\mathscr{S}_{1}\left(\mathscr{S}_{2}\right)$, the set of all subsets of $\{1, \cdots, n\}(\{1, \cdots, m\})$. Define the partial ordering by $\alpha$ $\leq \beta$ if $\alpha$ is a subset of $\beta$. Define $C_{1}\left(C_{2}\right)$ to consist of all states for process $1(2)$ which correspond to system failure. Since the systems are coherent $C_{1}$ and $C_{2}$ are increasing. Define $A_{1}\left(A_{2}\right)$ to be the infinitesimal generator for process 1(2). Then:

$$
A_{1}\left(\alpha, \alpha^{\prime}\right)= \begin{cases}\lambda_{i} ; & \alpha^{\prime}=\alpha+i, i \in \tilde{\alpha} \\ -\sum_{\tilde{\alpha}} \lambda_{i} ; & \alpha^{\prime}=\alpha \\ 0 ; & \text { elsewhere }\end{cases}
$$

with $A_{2}$ similarly defined.

Define a new Markov process with state space $\mathscr{I}_{1} \times \mathscr{S}_{2}$ by the infinitesimal generator:

$$
A\left((\alpha, \beta),\left(\alpha^{\prime}, \beta^{\prime}\right)\right)= \begin{cases}\lambda_{i} ; & \alpha^{\prime}=\alpha+i, i \in \tilde{\alpha}, \beta^{\prime}=\beta \\ \mu_{j} ; & \alpha^{\prime}=\alpha, \alpha \in C_{1}, \beta^{\prime}=\beta+j, j \in \tilde{\beta} \\ -\sum_{\tilde{\alpha}} \lambda_{i} ; & \alpha^{\prime}=\alpha, \beta^{\prime}=\beta, \alpha \in \widetilde{C}_{1} \\ -\left(\sum \tilde{\alpha} \lambda_{i}+\sum \widetilde{\beta} \mu_{j}\right) ; & \alpha^{\prime}=\alpha, \beta^{\prime}=\beta, \alpha \in C_{1} \\ 0 ; & \text { elsewhere }\end{cases}
$$

The Markov process with generator $A$ can be described by $\left\{\left(X_{1}(t), X_{2}(t)\right), t \geq 0\right\}$ where $X_{1}$ evolves according to $A_{1}$ while $X_{2}$ remains fixed at $X_{2}(0)$ until $X_{1}$ enters $C_{1}$. At that point $X_{1}$ and $X_{2}$ independently evolve governed by $A_{1}$ and $A_{2}$ respectively. The first passage time to $C_{1} \times C_{2}$ is thus the sum of $T_{1}$ and $T_{2}$ where $T_{1}$ and $T_{2}$ are independent. The Markov chain governed by $A$ under product partial ordering has monotone paths and its state space is a finite partially ordered set. Moreover, $C_{1} \times C_{2}$ is increasing. It follows from Corollary 1 that if the Markov chain is also stochastically monotone then $T_{1}+T_{2}$ (the first passage time to $C_{1} \times C_{2}$ ) is IFRA. By Corollary 1 it thus suffices to show that for $C$ increasing and $(\alpha, \beta) \leq\left(\alpha^{\prime}, \beta^{\prime}\right)$ with both $(\alpha, \beta)$ and $\left(\alpha^{\prime}, \beta^{\prime}\right)$ in $\widetilde{C}$ that:

$$
A((\alpha, \beta), C) \leq A\left(\left(\alpha^{\prime}, \beta^{\prime}\right), C\right) .
$$


Towards this end define $B_{1}=\{i:(\alpha+i, \beta) \in C\}, B_{2}=\{j:(\alpha, \beta+j) \in C\}, B_{1}^{\prime}=$ $\left\{i:\left(\alpha^{\prime}+i, \beta^{\prime}\right) \in C\right\}$, and $B_{2}^{\prime}=\left\{j:\left(\alpha^{\prime}, \beta^{\prime}+j\right) \in C\right\}$. Since $C$ is increasing it follows that $B_{1}$ $\subset B_{1}^{\prime}$ and $B_{2} \subset B_{2}^{\prime}$, and thus $\sum_{B_{1}} \lambda_{i} \leq \sum_{B_{1}^{\prime}} \lambda_{i}$ and $\sum_{B_{2}} \mu_{j} \leq \sum_{B_{2}^{\prime}} \mu_{j}$. Define

$$
H_{\alpha}=\left\{\begin{array}{lll}
1 & \text { if } & \alpha \in C_{1} \\
0 & \text { if } & \alpha \in \widetilde{C}_{1}
\end{array}\right.
$$

and similarly define $H_{\alpha^{\prime}}$. Since $\alpha \leq \alpha^{\prime}$ and $C_{1}$ is increasing it follows that $H_{\alpha} \leq H_{\alpha^{\prime}}$. Thus for $\left(\alpha^{\prime}, \beta^{\prime}\right) \in \widetilde{C}, A((\alpha, \beta), C)=\sum_{B_{1}} \lambda_{i}+\left(\sum_{B_{2}} \mu_{j}\right) H_{\alpha} \leq \sum_{B_{1}^{\prime}} \lambda_{i}+\left(\sum_{B_{2}^{\prime}} \mu_{j}\right) H_{\alpha^{\prime}}=A\left(\left(\alpha^{\prime}, \beta^{\prime}\right), C\right)$, so (11) is proved and $T_{1}+T_{2}$ is IFRA.

A similar argument proves the closure of IFRA distributions in discrete time. If $\boldsymbol{P}_{1}, \boldsymbol{P}_{2}$ are transition matrices for stochastically monotone Markov chains with monotone paths on partially ordered finite sets then a product space Markov chain is defined analogously as in (10) by:

$$
P\left((\alpha, \beta),\left(\alpha^{\prime}, \beta^{\prime}\right)\right)= \begin{cases}P_{1}\left(\alpha, \alpha^{\prime}\right) ; & \beta=\beta^{\prime}, \alpha \in \tilde{C}_{1} \\ P_{1}\left(\alpha, \alpha^{\prime}\right) P_{2}\left(\beta, \beta^{\prime}\right) ; & \alpha \in C_{1} \\ 0 ; & \text { elsewhere. }\end{cases}
$$

The Markov chain with transition matrix $P$ is of desired type and thus by Theorem 1 the first passage time to $C_{1} \times C_{2}$ (which is the sum of two independent IFRA distributions) is IFRA. Lemma 2 is then invoked and the result follows.

\section{Comments and additions.}

6.1. The following example shows that under the conditions of Theorem 1, the conclusion cannot be strengthened from IFRA to IFR even when the state space is totally ordered. Let $\left\{Y_{i}, i \geq 1\right\}$ be i.i.d. with $\operatorname{Pr}(Y=9)=\operatorname{Pr}(Y=2)=1 / 2$. Define $S_{n}=\sum_{1}^{n} Y_{i}, n \geq$ $1 ; S_{n}$ is a Markov process on the integers. It is stochastically monotone and has monotone paths. Consider, $T$, the first passage time to $\{10,11,12, \cdots\}$. Now $\operatorname{Pr}(T=2 \mid T \geq 2)=3 / 4$ while $\operatorname{Pr}(T=3 \mid T \geq 3)=1 / 2$; the distribution is not IFR. (A random variable $T$ is defined to be IFR if $\lambda_{k}=\operatorname{Pr}(T=k) / \operatorname{Pr}(T \geq k)$ is increasing.)

6.2. Consider a Markov chain on $[1,2, \ldots]$. Assume that the transition matrix is $T P_{2}$, i.e. that $P(i, j) P\left(i^{\prime}, j^{\prime}\right) \geq P\left(i, j^{\prime}\right) P\left(i^{\prime}, j\right)$ for all $i<i^{\prime}, j<j^{\prime}$. Lemma 3 below shows that the first passage time from state 1 to $C_{n}=\{i: i>n\}$ is IFR (increasing failure rate) for $n=1$, $2, \cdots$

Lemma 3. In a Markov chain with state space $\{1,2, \cdots\}$, and $T P_{2}$ transition matrix $P$, the first passage time $T\left(1, C_{n}\right)$, from state 1 to $C_{n}=\{i: i>n\}$ is IFR for $n=1,2, \cdots$.

Proof. Define $P_{k}(1, i)=\operatorname{Pr}\left(T\left(1, C_{n}\right)>k, X_{k}=i \mid X_{0}=1\right), i=1, \cdots, n$. Then $\lambda_{k}$, the failure rate for $T\left(1, C_{n}\right)$ at $k$ is given by:

$$
\lambda_{k}=\frac{\sum_{1}^{n} P_{k-1}(1, i) P\left(i, C_{n}\right)}{\sum_{1}^{n} P_{k-1}(1, i)}, \quad k=1,2, \cdots .
$$

Now, from (12):

$$
\lambda_{k+1}-\lambda_{k}=\frac{\sum_{1 \leq j<i \leq n}\left[P\left(i, C_{n}\right)-P\left(j, C_{n}\right)\right]\left[P_{k-1}(1, j) P_{k}(1, i)-P_{k-1}(1, i) P_{k}(1, j)\right]}{P_{k-1}\left(1, \widetilde{C}_{n}\right) P_{k}\left(1, \widetilde{C}_{n}\right)} .
$$

It follows from Theorem 2.2 of Karlin [10] that:

$$
P_{k-1}(1, j) P_{k}(1, i)-P_{k-1}(1, i) P_{k}(1, j) \geq 0, \text { for } j<i \text {. }
$$

Since $T P 2$ implies stochastic monotonicity (Keilson and Kester [12], Remark 1.1):

$$
P\left(i, C_{n}\right) \geq P\left(j, C_{n}\right) \text { for } i \geq j .
$$

Thus from (13), (14) and (15), $\lambda_{k}$ is increasing and $T\left(1, C_{n}\right)$ is thus IFR. $\square$ 
The above proof also shows that if for some $i_{0}, P(i, j)=0$ for all $i \geq i_{0}, j<i_{0}$ for the Markov chain defined in Lemma 3, then $T\left(i_{0}, C_{n}\right)$ is IFR for $n \geq i_{0}$. In particular if the Markov chain has monotone paths then the above holds for all $i_{0}$.

6.3. Consider a birth and death process with birthrates $\lambda_{i}, i \geq 0$ and death rates $\mu_{0}=0$, and $\mu_{i}, i \geq 1$. Using different methods Keilson [11] and Derman, Ross and Schechner [6] proved that the first passage time from 0 to $n$ is IFR. Below is another proof based on Lemma 3.

For convenience, set $\lambda_{n}=0$ and consider the state space to be $\{0, \ldots, n\}$, this modification having no bearing on the first passage time from 0 to $n$. Choose $c \geq$ $2 \max _{1 \leq i \leq n}\left(\lambda_{i}+\mu_{i}\right)$ and consider a Markov chain on $\{0, \cdots, n\}$ with transition matrix:

$$
P_{i, j}= \begin{cases}\lambda_{i} / c, & i=0, \cdots, n-1, j=i+1 \\ \mu_{i} / c, & i=1, \cdots, n, j=i-1 \\ 1-\left[\left(\lambda_{i}+\mu_{l}\right) / c\right], & i=0, \cdots, n, j=i \\ 0 & \text { elsewhere. }\end{cases}
$$

Our choice of $c$ insures that $P_{i, l} P_{l-1, i-1} \geq P_{i, l-1} P_{i-1, l}$, from which it follows that the transition matrix is TP2. By Lemma 3, the first passage time from 0 to $n$ for this discrete time chain is IFR. Next, have transitions in this Markov chain occur according to a Poisson process of rate $c$. The resulting continuous time process is the birth and death process under consideration. Since the discrete time first passage time is IFR it follows from the argument of Corollary 1 part (i) and exercise 7 page 98 of Barlow and Proschan [2], that the first passage time from 0 to $n$ for the continuous time process is IFR.

6.4. If the Markov chain is stochastically monotone but without monotone paths then the IFRA conclusion need not hold. For example in the following chain the first passage time from state 2 to state 3 is DFR (decreasing failure rate).

$$
P=\left(\begin{array}{ccc}
3 / 4 & 0 & 1 / 4 \\
1 / 2 & 0 & 1 / 2 \\
0 & 0 & 1
\end{array}\right)
$$

One might attribute the above example to the fact that starting in state 2 it is possible to go to state 1 which has smaller failure rate than state 2 (i.e. $P(1,3)<P(2,3)$ ). This might be eliminated if we require the chain to start in its smallest state. In the following example however the first passage time from state 1 to state 3 is not IFRA.

$$
P=\left(\begin{array}{lll}
.1 & .9 & 0 \\
.1 & 0 & .9 \\
0 & 0 & 1
\end{array}\right)
$$

Here $\operatorname{Pr}(T>2)=.19$ and $\operatorname{Pr}(T>3)=.109$, thus $\operatorname{Pr}^{2}(T>3)>\operatorname{Pr}^{3}(T>2)$ and consequently $T$ is not IFRA.

6.5. In the following example the Markov chain has monotone paths but is not stochastically monotone. The first passage time from state 1 to state 3 is DFR.

$$
\boldsymbol{P}=\left(\begin{array}{ccc}
0 & 1 / 2 & 1 / 2 \\
0 & 3 / 4 & 1 / 4 \\
0 & 0 & 1
\end{array}\right)
$$

6.6. Consider a stochastically monotone Markov chain whose state space is a partially ordered Polish space $E$, i.e. a complete separable metric space $E$ endowed with a closed partial ordering and with $\sigma$-algebra generated by the open sets. Assume also that there exists a point $a \in E$ with $a \leq x$ for all $x \in E$. Let $C$ be an increasing measurable set in $E$. Then $T(a, C)$, the first passage time from $a$ to $C$ is shown below to be NBU. A random 
variable on $\{0,1,2, \cdots\}$ is defined to be NBU if $\operatorname{Pr}(T \geq r+s) \leq \operatorname{Pr}(T \geq r) \operatorname{Pr}(T \geq s)$ for $r, s=1,2, \cdots$. The NBU property is weaker than IFRA.

To prove that $T(a, C)$ is NBU we first show that $T(x, C)$ is stochastically decreasing in $x$. This follows since given $x \leq y$, by Theorem 1 of Kamae, Krengel and O'Brien [9] we can construct two Markov chains $\left\{X_{n}, n \geq 0\right\}$ and $\left\{Y_{n}, n \geq 0\right\}$ on the same probability space, both governed by the transition law of the given Markov chain, with $X_{0}=x, Y_{0}=y$ and $X_{n} \leq Y_{n}$ a.s. for all $n$. Thus $T(x, C) \leq n$ implies $T(y, C) \leq n$ and $T(x, C)$ is thus stochastically larger than $T(y, C)$.

It follows that $T(a, C)$ is stochastically larger than $T(x, C)$ for all $x \in E$. Thus $T(a, C)$ is stochastically larger than $T\left(X_{r}^{*}, C\right)$ where $X_{r}^{*}$ is an $E$ valued random variable whose distribution is the conditional distribution of $X_{r}$ given that $X_{0}=a$ and $T(a, C) \geq r$. Thus $\operatorname{Pr}(T(a, C) \geq r+s)=\operatorname{Pr}(T(a, C) \geq r) \operatorname{Pr}\left(T\left(X_{r}, C\right) \geq s \mid T(a, C) \geq r\right)=\operatorname{Pr}(T(a, C) \geq$ $r) \operatorname{Pr}\left(T\left(X_{r}^{*}, C\right) \geq s\right) \leq \operatorname{Pr}(T(a, C) \geq r) \operatorname{Pr}(T(a, C) \geq s)$, and $T(a, C)$ is thus NBU.

6.7. The definition of IFRA for discrete distributions followed here (Section 2) is the same as in Barlow-Proschan [1], page 94, and Block and Savits [4]. Ross, Shashahani and Weiss [13] use the term SSLSF (star-shaped log survival function) for what we call IFRA, and reserve the term IFRA for the weaker property, $(1 / k) \sum_{i=1}^{k} \operatorname{Pr}(T=i \mid T \geq i)$ increasing.

\section{APPENDIX}

Proof of Lemma 1. Suppose first that $f$ is constant over $D$. Define a point $x$ to be maximal in a partially ordered set $A$ if $x \in A$ and for all $y \in A$ distinct from $x, y \geq x(y$ is not bigger than $x$ under the partial ordering). Clearly every finite partially ordered set has at least one maximal point. Take a maximal point of $D$ and label it $d_{1}$; then take a maximal point of $D-d_{1}$ and label it $d_{2}$. Continue this process until one point remains and label it $d_{m}$. For $i<j$, since $d_{i}$ is maximal in a set containing $d_{j}$, it follows that $d_{i} \not \leq d_{j}$.

Next, consider a general decreasing function $f$ over $D$. Let $\alpha_{1}<\alpha_{2}<\ldots<\alpha_{k}$ denote the distinct values of $f(x)$ as $x$ ranges over $D$. Define $A_{i}=\left\{x: f(x)=\alpha_{i}\right\}$, set $n_{i}$ equal to the number of elements in $A_{i}, m_{1}=0$ and $m_{i}=\sum_{1}^{i-1} n_{j}, i=2, \cdots, k$. Using the above result for constant $f$ label the points in $A_{i}$ as $d_{m_{l}+1}, \cdots, d_{m_{l+1}}$, in such a way that $m_{i}+1 \leq r<s \leq$ $m_{i+1}$ implies $d_{r} \not d_{s}$.

This provides a labeling of the points in $D$ which clearly has the desired properties.

\section{REFERENCES}

[1] Barlow, R. E. and Proschan, F. (1975). Statistical Theory of Reliability and Life Testing. Holt, Rhinehart and Winston, New York.

[2] Birnbaum, Z. W., Esary, J. D., and Marshall, A. W. (1966). Stochastic characterization of wearout for components and systems. Ann. Math. Statist. 37 816-825.

[3] Block, H. and Savits, T. (1976). The IFRA closure problem. Ann. Probab. 4 1030-1033.

[4] Cinlar, E. (1975). Introduction to Stochastic Processes. Prentice Hall, Englewood Cliffs, New Jersey.

[5] Daley, D. J. (1968). Stochastically monotone Markov chains. Z. Wahrsch. verw. Gebiete 10 305-317.

[6] Derman, C., Ross., S. M., and Schechner, Z. (1979). A note on first passage times in birth and death and negative diffusion processes. Unpublished manuscript.

[7] Esary, J. D., Marshall, A. W., and Proschan, F. (1973). Shock models and wear processes. Ann. Probab. 1 627-649.

[8] Feller, W. (1957). An Introduction to Probability Theory and its Applications, Vol. 1. Wiley, New York.

[9] Kamae, T., Krengel, U., and O’Brien, G. C. (1977). Stochastic inequalities on partially ordered spaces. Ann. Probab. 5 899-912.

[10] Karlin, S. (1964). Total positivity, absorption probabilities and applications. Trans. Amer. Math. Soc. 111 33-107.

[11] KeILson, J. (1979). Markov Chain Models-Rarity and Exponentiality. Springer-Verlag, New York.

[12] Keilson, J. and Kester, A. (1977). Monotone matrices and monotone Markov processes. Stochastic Process. Appl. 5 231-241. 
[13] Ross, S. M., Shashahani, M. and Weiss, G. (1979). On the number of component failures in systems whose component lives are exchangeable. Unpublished manuscript.

[14] Rota, G.-C. (1964). On the foundations of combinatorial theory I. Theory of Mobius functions. Z. Wahrsch. verw. Gebiete 2 340-368.

Mathematics Department

City College, CUNY

NEW YORK, NEW YoRK 10031
Mathematics Department

OLD DOMINION UNIVERSITY

NORFOLK, VIRGINIA 23508 